\title{
Prenatal Diagnosis of Walker-Warburg Syndrome Using Single Nucleotide Polymorphism Array: A Clinical Experience from Three Related Palestinian Families with Congenital Hydrocephalus
}

\author{
Iman S. Abumansour, MBBS ${ }^{1,2}$ Eman Al Sulmi, MBBS, FRCSC ${ }^{3}$ \\ Bernard N. Chodirker, MD, FCCMG, FRCPC ${ }^{1,2}$ Jennifer C. Hunt, MD, FRCSC ${ }^{3}$
}

\footnotetext{
${ }^{1}$ Department of Pediatrics and Child Health, University of Manitoba, Winnipeg, Manitoba, Canada

${ }^{2}$ Department of Biochemistry and Medical Genetics, University of Manitoba, Winnipeg, Manitoba, Canada

${ }^{3}$ Section of Maternal Fetal Medicine, Department of Obstetrics, Gynecology and Reproductive Sciences, University of Manitoba, Winnipeg, Manitoba, Canada
}

Am J Perinatol Rep 2015;5:e116-e120.

\begin{abstract}
Address for correspondence Iman S. Abumansour, MBBS, Department of Biochemistry and Medical Genetics, University of Manitoba, FE229, 820 Sherbrook Street, Winnipeg, Manitoba, R3A 1R9, Canada (e-mail: iabumansour@exchange.hsc.mb.ca).
\end{abstract}

\begin{abstract}
Keywords

- Walker-Warburg syndrome

- congenital hydrocephalus

- prenatal SNP array

- syndromic hydrocephalus

Background Congenital hydrocephalus is a common and often disabling disorder. Various syndromic forms of hydrocephalus have been reported in the Palestinian population including Walker-Warburg syndrome (WWS), Carpenter syndrome, and Meckel syndrome.

Aim In this report we discuss the antenatal diagnosis of congenital hydrocephalus in three related Palestinian families.

Method Single nucleotide polymorphism (SNP) array was performed prenatally for the third affected fetus.

Results A diagnosis of WWS was found and molecular testing revealed a known pathogenic mutation in the POMT2 gene. An affected fetus from the other family was diagnosed and tested postnatally in light of this finding. Testing of another affected stillborn offspring was performed and revealed the same mutation.

Conclusions Here, we show that the use of prenatal SNP array testing can be helpful in elucidating the etiology of congenital hydrocephalus and in guiding appropriate perinatal care. Also, testing for this specific POMT2 mutation should be considered in cases of prenatally detected hydrocephalus in Palestinian families.
\end{abstract}

Congenital hydrocephalus is a common and often disabling disorder that represents approximately $50 \%$ of all forms of hydrocephalus. ${ }^{1}$ Antenatal diagnosis of congenital hydrocephalus is made with an ultrasound finding of fetal ventriculomegaly that exceeds $10 \mathrm{~mm}$ at any gestational age. ${ }^{2}$
Syndromic congenital hydrocephalus is defined as hydrocephalus noted in conjunction with at least one other major abnormality or three minor congenital anomalies. ${ }^{1}$ The most frequent chromosomal abnormalities causing syndromic hydrocephalus are mosaic trisomy 9 , trisomy 9 p, trisomy 13 , received

September 28, 2014 accepted after revision February 28, 2015 published online April 27, 2015
DOI http://dx.doi.org/ 10.1055/s-0035-1549298. ISSN 2157-7005.
Copyright $\odot 2015$ by Thieme Medical Publishers, Inc., 333 Seventh Avenue, New York, NY 10001, USA. Tel: +1(212) 584-4662.
License terms

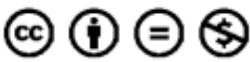


trisomy 18 , mosaic triploidy, and partial $6 \mathrm{p}$ terminal deletion. ${ }^{1} \mathrm{X}$-linked congenital hydrocephalus is also seen such as in L1CAM-related syndromes. However, autosomal recessive single gene disorders, such as dystroglycanopathies, including Walker-Warburg (WWS) syndrome remain the main mode of inheritance for congenital hydrocephalus. ${ }^{1}$ The associated features of syndromic congenital hydrocephalus can help in identifying a particular genetic syndrome. This can be difficult antenatally as many of the associated features are unobservable on fetal ultrasound. Therefore, the prenatal diagnosis of a specific syndromic congenital hydrocephalus is a diagnostic dilemma particularly when conventional chromosomal analysis is normal.

We report three cases with antenatal diagnoses of congenital hydrocephalus from related Palestinian families. The couples were related via the male partners (two are brothers and the third is their first cousin) and each mating was consanguineous. The diagnosis was achieved prenatally in one family and that was helpful in providing counselling for the other families.

\section{Case Series}

Three pregnant women were seen in the fetal assessment unit at a tertiary care hospital within the same year with antenatal diagnosis of fetal hydrocephalus. Interestingly, the three families were identified by their similar surnames upon their visits to the unit.

\section{Patient A}

A 33 year-old G5P2 female patient presented at 11 weeks of gestation to the genetics department with a history of termination of pregnancy due to fetal hydrocephalus and a suspected Dandy-Walker malformation. In the subsequent pregnancy, a miscarriage at 16 weeks gestation occurred and the fetus had similar findings. She had not been assessed by the genetics department during that pregnancy. The current pregnancy was initially unremarkable. She denied any maternal illnesses or exposure to teratogens. At 13 weeks and 4 days of gestation the nuchal translucency was $2.2 \mathrm{~mm}$ and there was evidence of a large subchorionic hemorrhage. A repeat ultrasound at 16 weeks and 5 days of gestation showed frontal bone scalloping. Her modified integrated prenatal screen for aneuploidy (first trimester nuchal translucency and second trimester serum quadruple test) was negative. At 19 weeks and 5 days of gestation, bilateral ventriculomegaly and suspected cerebellar hypoplasia were noted on fetal ultrasound. The measurements of lateral ventricle dilatation were not provided. Karyotype by amniotic fluid was normal $(46, \mathrm{XX})$. The family was counselled that the hydrocephalus was most likely secondary to an autosomal recessive disorder given the family history of consanguinity; the two previously affected pregnancies; and paternal aunt with similar presentation (-Fig. 1). The couple opted for expectant management. The fetus was born via cesarean delivery after the presentation of the other cases.

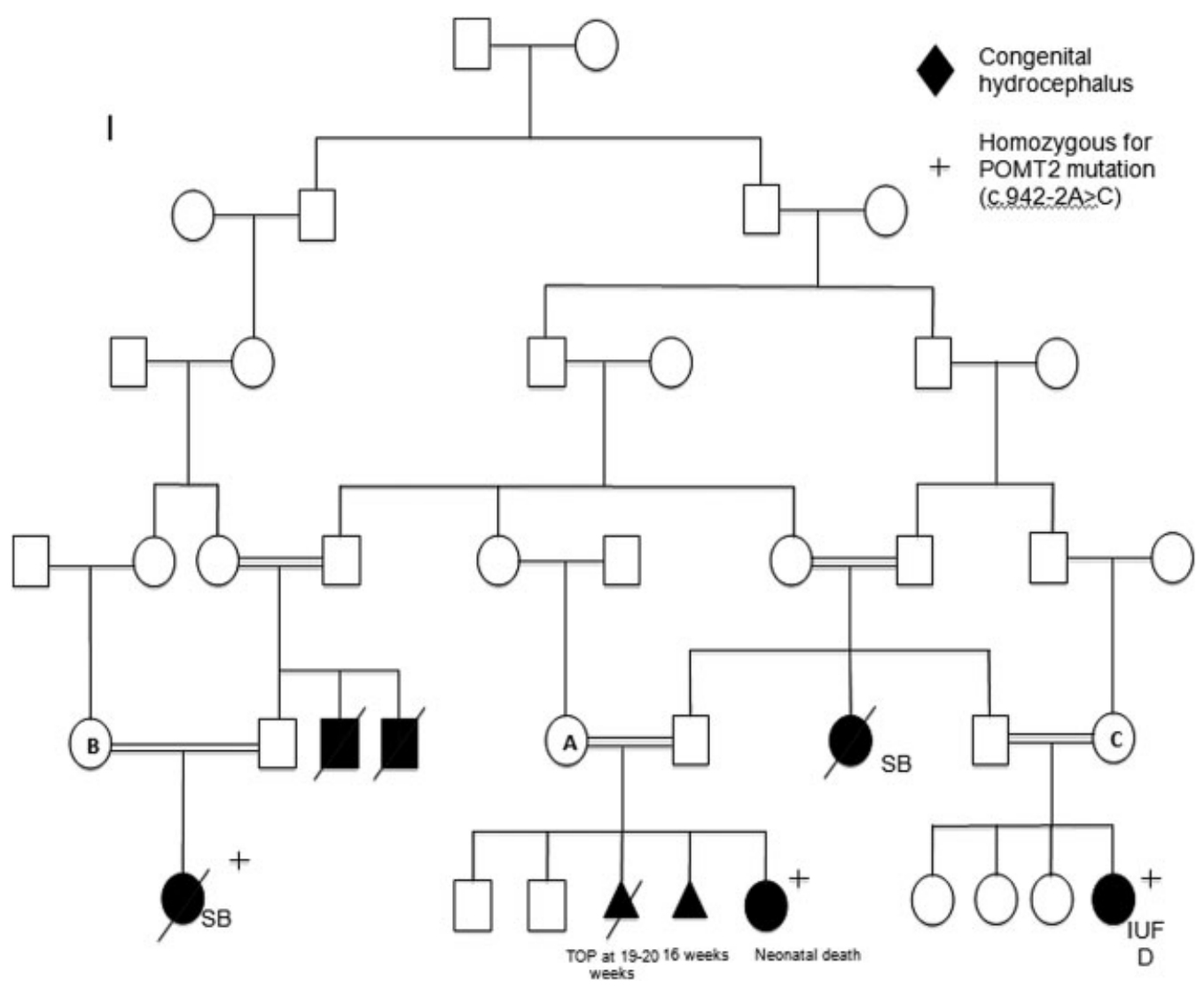

Fig. 1 A family pedigree connected the three cases. SB, stillbirth; TOP, termination of pregnancy; IUFD, intrauterine fetal death. 


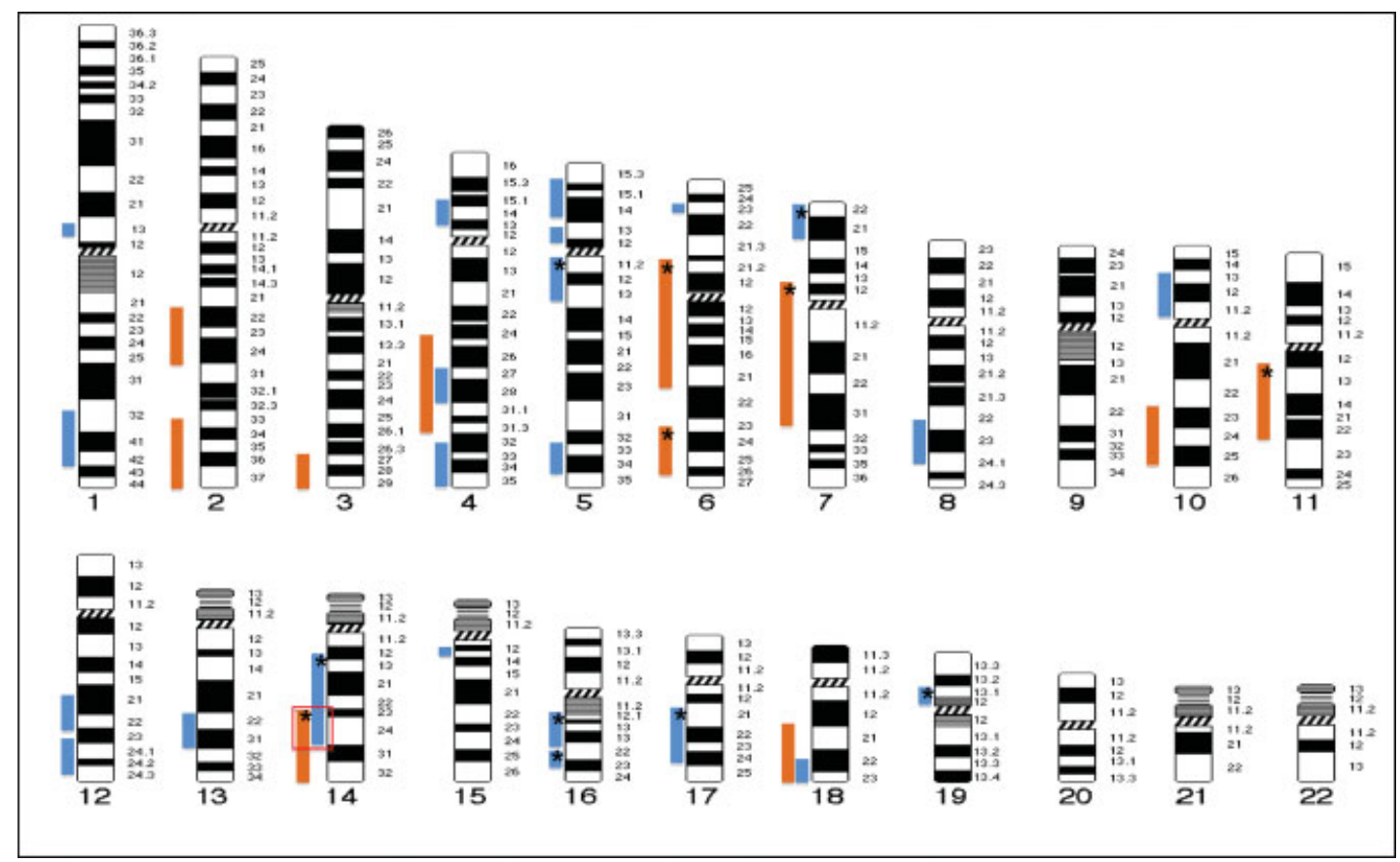

Fig. 2 An idiogram showed the regions of homozygosity found in the infant of patient A (blue bars) and the fetus of patient $C$ (orange bars). Red square indicated the shared region of homozygosity (14q23.1-q24.3) that harbored POMT2 gene. *, regions that contained genes linked to hydrocephalus.

\section{Patient B}

A 24 year-old G1P0 female patient presented at 21 weeks of gestation with fetal hydrocephalus on ultrasound. The lateral ventricles were dilated up to $21 \mathrm{~mm}$ bilaterally. The patient reported a 3-day history of bleeding earlier in the pregnancy; otherwise, she denied any maternal illnesses or exposure to teratogens. Maternal serum screening was negative. The family history was significant for paternal uncles who died during the neonatal period due to congenital hydrocephalus. Amniocentesis was done for chromosomal analysis and DNA banking. The karyotype was normal $(46, \mathrm{XX})$. This couple also opted for expectant management. A stillborn female was delivered via elective cesarean delivery at gestational age of 37 weeks due to severe hydrocephalus and macrocephaly. Autopsy was declined. The cesarean delivery was complicated by severe postpartum hemorrhage requiring ligation of the uterine arteries.

\section{Patient C}

A 26 year-old G4P3 female patient presented at 28 weeks of gestation with similar findings of fetal ventriculomegaly of $27 \mathrm{~mm}$ bilaterally, and suspected cerebellar hypoplasia on ultrasound. The pregnancy history was unremarkable. Maternal serum screening was not done. Amniocentesis was performed for chromosomal analysis, DNA banking, and single nucleotide polymorphism (SNP) array. The karyotype was normal $(46, \mathrm{XX})$. The family decided to carry on with pregnancy. As the pregnancy progressed, the fetus developed worsening hydrocephalus and subsequent macrocephaly on follow-up ultrasound scans. As the prognosis was expected to be poor, the family opted for neonatal palliative care after delivery. At 35 weeks and 6 days of gestation, the fetal head circumference was above the 90th centile for gestational age. Fetal cephalocentesis was performed to facilitate vaginal delivery. The procedure was complicated by an intrauterine fetal death 2 days later. A medical induction of labor was initiated and a stillborn female infant was delivered vaginally at gestational age of 36 weeks and 2 days. Right microphthalmia was noted at the time of delivery. An autopsy was declined.

\section{Results}

Prenatal SNP array results were eventually obtained. These revealed multiple regions of homozygosity (ROH) (-Fig. 2). Analysis of the ROH was done using Genomic oligoarray and SNP array evaluation tool v2.0 (A tool developed in a collaborative effort between the Center for Computational Science at University of Miami, Coral Gables, Florida and the Section of Genetics in the Department of Pediatrics at Oklahoma University Health Sciences Center, Oklahoma City, OK). It identified $5 \mathrm{ROH}$ out of 11 regions where hydrocephalus-associated genes were annotated (-Fig. $\mathbf{2}$ ). The genes within the indicated regions that were suspicious to be the etiology of the hydrocephalus included: LAMB1 (lissencephaly 5; online Mendelian inheritance in man [OMIM] \#615191), B3GNT1 (muscular dystrophy-dystroglycanopathy type A; OMIM \#615287), FLVCR2 (proliferative vasculopathy and hydranencephaly-hydrocephaly syndrome; OMIM \#225790), POMT2 (muscular dystrophy-dystroglycanopathy type A, B, and C; OMIM \#613150, 613156, and 613158, respectively), and CCDC88C (nonsyndromic autosomal recessive hydrocephalus; OMIM \#236600). A review of the literature revealed that WWS has been seen previously in Palestinian populations. ${ }^{3} \mathrm{~A}$ 

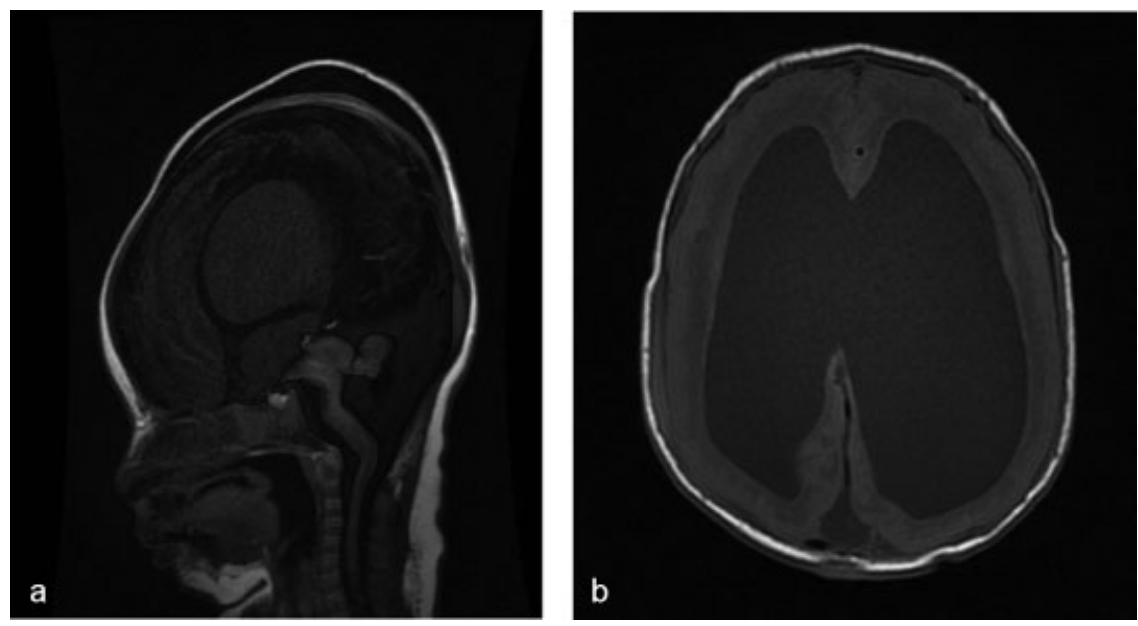

Fig. 3 (a) Sagittal T1 showed severely hypoplastic pons and cerebellar hemispheres consistent with Walker-Warburg syndrome. (b) Axial T1 showed cobblestone lissencephaly of both the cerebral hemispheres.

previously reported case of WWS in a consanguineous Palestinian family was found to be associated with a POMT2 mutation (c.924-2 A > C). ${ }^{4}$ Given those reports and the SNP array results, molecular testing was requested for this particular mutation in POMT2 gene from the banked DNA obtained on the fetus of patient $C$.

While molecular testing result for patient $C$ was pending, patient $\mathrm{A}$ delivered a female infant at 42 weeks 2 days gestation via cesarean delivery for fetal distress. Apgar scores 1 and 7 in 1 and 5 minutes, respectively. Birth weight was $3,620 \mathrm{~g}$. The infant required nasal continuous positive airway pressure. Dysmorphic features were noted including frontal bossing, a depressed nasal bridge, and bilateral eye proptosis. She had significant hypotonia axially and peripherally predominantly in the upper limbs. Deep tendon reflexes were absent. A significantly elevated creatine kinase level was found $(5,600 \mathrm{U} / \mathrm{L})$. Brain magnetic resonance imaging revealed cobblestone lissencephaly with cerebellar hypoplasia and hypoplastic brain stem in a " $z$ "-shaped configuration on the sagittal plane (-Fig. 3 ). These findings were consistent with a clinical diagnosis of WWS. The infant was placed on palliative care and died a few days after discharge from the neonatal intensive care unit. Postnatal SNP array on this infant revealed $22 \mathrm{ROH}, 6$ of which had hydrocephalus-

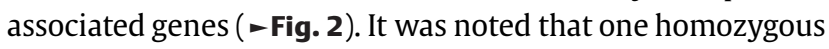
region on chromosome 14 (20 $\mathrm{Mb}$ in size) was shared between the infant of patient $A$ and the fetus of patient $C$. This region was found to harbor the POMT2 gene; therefore, molecular testing was requested for this infant as well. The results revealed that infant of patient $\mathrm{A}$ and the fetus of patient $C$ were homozygous for the previously described Palestinian mutation (c.924-2 A > C). Molecular testing was eventually performed on the fetus of patient $B$ and as expected it was positive for the same mutation ( $\mathbf{- F i g . ~} \mathbf{1})$.

\section{Discussion}

Prenatal SNP array in consanguineous families is a useful tool in identifying anomalous fetuses at risk for ethnic-specific autosomal recessive disorders. ${ }^{5-8}$ Briefly, SNP array is a cytogenetic technique based on genomic comparative hybridization using commercially available platforms containing SNPs probes. The test can detect unbalanced chromosomal abnormalities with a higher resolution to reveal a submicroscopic copy number variation (gain or loss). ${ }^{9}$ It can identify aneuploidy and triploidy. ${ }^{6}$ In addition, SNP array identifies $\mathrm{ROH}$ that may indicate ancestral homozygosity, parental consanguinity or uniparental disomy. ${ }^{8}$ There is no doubt in its utility to direct the clinical diagnosis particularly in prenatal setting after ultrasound detection of fetal abnormalities. $^{6,7,9}$ It is a relatively fast diagnostic test especially in time-sensitive situations such as termination of pregnancy. However, this test has limitations. It cannot detect balanced chromosomal aberrations. Further, it cannot reveal the location of the extrachromosomal material. ${ }^{9}$

For patient C, prenatal SNP array was a helpful tool in achieving the diagnosis of WWS antenatally and in providing appropriate counselling and obstetrical management. At the time of presentation of patients $A$ and $B$, the underlying etiology of congenital hydrocephalus and its prognosis were not initially apparent and the differential diagnosis was extensive. We could not do prenatal SNP array on them due to budget limitations. For that, it was difficult to counsel these families with respect to the prognosis and options for prenatal and postnatal management. It is possible that cesarean delivery may have been avoided in these cases if the diagnosis and prognosis had been ascertained before delivery. Also, the option of neonatal palliative care could have been considered prenatally for the infant of patient $A$ and unnecessary neonatal resuscitation could have been avoided.

WWS is a genetically heterogeneous autosomal recessive disorder. It is the most severe phenotype of dystroglycanopathies. ${ }^{10}$ It is characterized by congenital muscular dystrophy, cobblestone lissencephaly, and ocular malformations. ${ }^{11,12}$ There is a wide spectrum of ocular manifestations in WWS. These include microphthalmia, anterior segment anomalies, such as Peters anomaly, glaucoma and cataracts, optic nerve coloboma or hypoplasia, and retinal dysplasia. ${ }^{13}$ A case of 
WWS was reported where retinal dysplasia and echogenic lenses were detected prenatally in second trimester with detailed ultrasound of fetal eyes. ${ }^{14}$ However, this was not visualized in our cases to confirm the diagnosis of WWS prenatally. Molecular confirmation of WWS in those consanguineous families is required to determine recurrence risk in future pregnancies in addition to providing carrier tests to relatives at risk. In genetically heterogeneous autosomal recessive conditions, SNP array aids in the selection of candidate genes within the homozygous regions for sequence or targeted mutation analysis.

\section{Conclusion}

The use of prenatal SNP array testing can be very helpful in elucidating the etiology of autosomal recessive disorders manifesting in utero. Prenatal diagnosis can then allow appropriate patient counselling regarding prognosis as well as obstetrical and neonatal care. Testing for this specific POMT2 mutation (c.924-2 A > C) should be considered in cases of prenatally detected hydrocephalus in affected fetuses with parents of Palestinian descent.

\section{Acknowledgment}

The cases were seen in Health Sciences Centre in Winnipeg, Canada. The Diagnostic Services of Manitoba, which is represented by cytogenetics and molecular laboratories, supported the send-out SNP array and molecular tests. Many thanks are to the families who gave the permission to publish the cases.

\section{References}

1 Verhagen JM, Schrander-Stumpel CT, Krapels IP, et al. Congenital hydrocephalus in clinical practice: a genetic diagnostic approach. Eur J Med Genet 2011;54(6):e542-e547
2 Cavalheiro S, Moron AF, Almodin CG, et al. Fetal hydrocephalus. Childs Nerv Syst 2011;27(10):1575-1583

3 Zlotogora J. Genetic disorders among Palestinian Arabs. 2. Hydrocephalus and neural tube defects. Am J Med Genet 1997;71(1): 33-35

4 Manzini MC, Gleason D, Chang BS, et al. Ethnically diverse causes of Walker-Warburg syndrome (WWS): FCMD mutations are a more common cause of WWS outside of the Middle East. Hum Mutat 2008;29(11):E231-E241

5 Liao C, Fu F, Li R, et al. Implementation of high-resolution SNP arrays in the investigation of fetuses with ultrasound malformations: 5 years of clinical experience. Clin Genet 2014;86(3): 264-269

6 Srebniak MI, Boter M, Oudesluijs GO, et al. Genomic SNP array as a gold standard for prenatal diagnosis of foetal ultrasound abnormalities. Mol Cytogenet 2012;5(1):14

7 Srebniak M, Boter M, Oudesluijs G, et al. Application of SNP array for rapid prenatal diagnosis: implementation, genetic counselling and diagnostic flow. Eur J Hum Genet 2011;19(12): 1230-1237

8 Sund KL, Zimmerman SL, Thomas C, et al. Regions of homozygosity identified by SNP microarray analysis aid in the diagnosis of autosomal recessive disease and incidentally detect parental blood relationships. Genet Med 2013;15(1):70-78

9 Srebniak MI, Mout L, Van Opstal D, Galjaard R-JH. 0.5 Mb array as a first-line prenatal cytogenetic test in cases without ultrasound abnormalities and its implementation in clinical practice. Hum Mutat 2013;34(9):1298-1303

10 Devisme L, Bouchet C, Gonzalès $\mathrm{M}$, et al. Cobblestone lissencephaly: neuropathological subtypes and correlations with genes of dystroglycanopathies. Brain 2012;135(Pt 2):469-482

11 Dobyns WB, Pagon RA, Armstrong D, et al. Diagnostic criteria for Walker-Warburg syndrome. Am J Med Genet 1989;32(2): 195-210

12 Murphy KJ, PeBenito R, Storm RL, Ferretti C, Liu DP. WalkerWarburg syndrome. Case report and literature review. Ophthalmic Paediatr Genet 1990;11(2):103-108

13 Gerding H, Gullotta F, Kuchelmeister K, Busse H. Ocular findings in Walker-Warburg syndrome. Childs Nerv Syst 1993;9(7):418-420

14 Brasseur-Daudruy M, Vivier PH, Ickowicz V, Eurin D, Verspyck E. Walker-Warburg syndrome diagnosed by findings of typical ocular abnormalities on prenatal ultrasound. Pediatr Radiol 2012; 42(4):488-490 